Journal for ImmunoTherapy of Cancer

To cite: Gatti-Mays M, Gulley JL. Real-world insights on preferred treatments for steroid-refractory immune checkpoint inhibitor-induced pneumonitis. Journal for ImmunoTherapy of Cancer 2021;9:e002252. doi:10.1136/ jitc-2020-002252

Accepted 12 January 2021

\section{Linked}

- http://dx.doi.org/10.1136/ jitc-2020-001884

- http://dx.doi.org/10.1136/ jitc-2020-001731

Check for updates

${ }^{1}$ Pelotonia Institute for ImmunoOncology, Division of Medical Oncology, The Ohio State University Comprehensive Cancer Center, Columbus, $\mathrm{OH}$, USA

${ }^{2}$ Genitourinary Malignancy Branch, National Cancer Institute, Bethesda, Maryland, USA

\section{Correspondence to} Dr Margaret Gatti-Mays; margaret.gatti-mays@osumc. edu

\title{
Real-world insights on preferred treatments for steroid-refractory immune checkpoint inhibitor-induced pneumonitis
}

\author{
Margaret Gatti-Mays (D) , ${ }^{1}$ James L Gulley (D) ${ }^{2}$
}

Immune checkpoint inhibitor (ICI)-induced pneumonitis has been considered a rare immune-related adverse event (irAE). Incidence is estimated at less than $5 \%$ of clinical trial patients, with high-grade pneumonitis occurring in less than $1 \%$ of patients who receive ICIs. ${ }^{12}$ However, as ICIs gain traction as standard therapies, real-world data suggest a slightly higher incidence than is seen in clinical trial populations, with ICI-induced pneumonitis occurring in up to $19 \%$ of patients. The risk of developing ICI-induced pneumonitis varies among ICI classes. Antiprogrammed cell death 1 (PD-1) inhibitors have a higher incidence than anti-PD-L1 inhibitors or anti-cytotoxic T-lymphocyteassociated protein 4 (CTLA-4) inhibitors, and combination therapy results in a higher risk of pneumonitis compared with ICI monotherapy. ${ }^{3}$ In addition, ICI-induced pneumonitis is most common in patients with lung cancer and is the most common anti-PD-1/ L1-related cause of death across all tumor types. $^{24}$

ICI-induced pneumonitis generally develops within the first 3 months of treatment. ${ }^{5}$ High-dose steroids such as prednisone/methylprednisolone $1-2 \mathrm{mg} / \mathrm{kg} /$ day are used initially. If patients do not improve after 48-72 hours on high-dose steroids, they are classified as steroid refractory. Current consensus guidelines by National Comprehensive Cancer Network (NCCN), ${ }^{6}$ Society for Immunotherapy of Cancer, ${ }^{1}$ and European Society for Medical Oncology (ESMO) ${ }^{7}$ recommend escalating immunosuppression with the addition of one of the following agents to high-dose steroids for steroidrefractory irAEs: (1) infliximab $5 \mathrm{mg} / \mathrm{kg}$, (2) cyclosporine $2.5 \mathrm{mg} / \mathrm{kg} /$ day in divided doses, (3) mycophenolate mofetil 1-1.5 g two times a day, (4) intravenous immunoglobulin (IVIG)
$2 \mathrm{~g} / \mathrm{kg}$ in divided doses over $2-5$ days, or (5) tocilizumab $0.6 \mathrm{mg} / \mathrm{kg}$. However, consensus guidelines do not specify the preferred initial agent, the optimal order of agents, or the optimal number of doses. The tumor necrosis factor (TNF)- $\alpha$ inhibitor infliximab is often the preferred first option as it has been used successfully in other irAEs such as colitis, but there are little published data on its efficacy in treating steroid-refractory ICI-induced pneumonitis. 8

Balaji et $a l^{\hat{\theta}}$ and Beattie et $a l^{10}$ present the largest retrospective chart reviews to date ( $n=12$ and 26 patients, respectively) on the clinical management and course of patients at their respective institutions with steroidrefractory ICI-induced pneumonitis. Given the low incidence of pneumonitis and the even lower incidence of steroid-refractory pneumonitis, these retrospective reviews provide an important clinical insight into the efficacy of recommended therapies (specifically infliximab) and long-term clinical outcomes of patients with steroid-refractory ICI-induced pneumonitis.

In both studies, most patients who developed ICI-induced pneumonitis had lung cancer, which is consistent with prior studies. ${ }^{2}$ It is not surprising that at least $75 \%$ of patients were current or former smokers and many had prior chest radiation. However, only a small number had clinically evident preexisting pulmonary conditions. Hypotheses about increased incidence of pneumonitis in patients with lung cancer who receive ICI include greater tumor burden in the lungs than other cancers, pre-existing lung conditions, and prior pulmonary damage from tobacco use. While this causal association has previously been documented, it is important to note that tobacco-associated lung cancers generally have a higher tumor mutational 
burden, higher PD-L1 expression and respond better to ICIs. The retrospective studies presented here raise important questions about the true risk of current or prior tobacco use and the development of pneumonitis. This causal association has yet to be verified in prospective studies. Additional studies needed to better identify which patients are at higher risk of developing pneumonitis, specifically primary steroid-refractory ICI-induced pneumonitis.

Balaji et aldescribe the clinical management and disease course of 12 patients with primary steroid-refractory ICIinduced pneumonitis (defined by the authors as lack of clinical improvement after at least 48 hours and up to 14 days of high-dose corticosteroids). The identified patients were referred to the multidisciplinary immunerelated toxicity team or pulmonary outpatient clinic with ICI-induced pneumonitis. In this study, primary steroid refractoriness occurred after a median of 5 doses of ICI, resulting in grade $2(n=3)$ and grade $3(n=9)$ symptomatic pneumonitis. The most commonly identified radiographic pattern was diffuse alveolar damage. The mean dose of steroids administered was prednisone equivalent $75 \mathrm{mg}$ /day prior to receiving additional immunosuppressive therapy. Clinical management included treatment with IVIG $(\mathrm{n}=7$; started a mean of 17 days after steroid initiation), infliximab ( $\mathrm{n}=2$; started 7 and 8 days after steroid initiation), and combination IVIG + infliximab $(n=3$; infliximab added to IVIG on days 8, 9, and 15). Almost all patients with primary steroid-refractory ICI-induced pneumonitis required management in the intensive care unit; around half required mechanical ventilation. The authors report that $75 \%$ of patients in this study died of steroid-refractory ICI-induced pneumonitis or infectious complications, including all five patients treated with infliximab (two as monotherapy, three in combination with IVIG).

Beattie et al describe the clinical course of 26 patients who had either primary steroid-refractory ICI-induced pneumonitis ( $\mathrm{n}=12$; defined as no improvement or worsening of pneumonitis with initial high-dose steroid monotherapy) or steroid-resistant ICI-induced pneumonitis ( $\mathrm{n}=14$; defined as initial response to steroid with subsequent recurrence of pneumonitis during steroid tapering in the absence of ICI rechallenge). The authors searched for administration of immunomodulatory agents and evaluated the clinical management and course for patients who clinically and/or pathologically had ICI-induced pneumonitis. All patients with primary steroid-refractory ICI-induced pneumonitis were treated with at least 60 $\mathrm{mg}$ of prednisone equivalent. Median time to addition of an immunomodulator for primary steroid-refractory ICI-induced pneumonitis was 7 days, and median time to additional treatment for steroid-resistant pneumonitis during steroid taper was 2.9 months. The study reported more severe disease in patients with primary steroidrefractory ICI-induced pneumonitis (grade $2=1$, grade $3, \mathrm{n}=9$; grade $4, \mathrm{n}=2$ ) than in patients with resistant pneumonitis (grade 2, $\mathrm{n}=10$; grade $3, \mathrm{n}=4$; grade $4, \mathrm{n}=0$ ). Most of the retrospective study included patients treated with infliximab monotherapy $(\mathrm{n}=15 ; 58 \%)$, mycophenolate mofetil monotherapy $(n=5 ; 19 \%)$ or infliximab comination therapy $(\mathrm{n}=5 ; 19 \%)$. However, further investigation into the subgroups shows that all patients with primary steroid-refractory pneumonitis $(\mathrm{n}=12)$ received infliximab as monotherapy or in combination with mycophenolate, and most patients with recurrent pneumonitis received infliximab, and/or mycophenolate. Of the 26 patients identified, more than half $(62 \%)$ were hospitalized and, despite aggressive treatment, only 10 had a durable improvement following additional immunosuppression plus steroids. However, when focusing on the primary steroid-refractory patients, only $3 / 12(25 \%)$ had durable desponses to combination immunosuppression and all 12 patients died with cause of death attritbued to pneumonitis or infectious complications in half of these patients (See supplemental table 3).

Steroid-refractory ICI-induced pnemonitis is a rare irAE with a poor prognosis. Patients with primary steroidrefractory ICI-induced pneumonitis have a high all-cause mortality rate (pneumonitis or infectious complications) and a worse prognosis than patients with recurrent ICI-induced pneumonitis. Identifying ICI-induced pneumonitis may be challenging due to varying clinical presentations, but the most common radiographic pattern is diffuse aveolar damage. It is unclear if rapid escalation from steroid monotherapy to combination therapy with other immune modulators can fully reverse the disease course or improve clinical outcomes in patients with high-grade primary steroid-refractory ICI-induced pneumonitis. However, therapy escalation may lead to durable improvement (although rarely complete resolution) in steroid-refractory ICI-induced pneumonitis in up to $30 \%-40 \%$ of patients. Prospective studies on the efficacy of immune modulators or combinations are greatly needed for primary steroid-refractory ICI-induced pneumonitis. Furthermore, in addition to standard endpoints like 90-day morbidity and duration of immunosuppression, these trials should also incorporate clinically meaningful endpoints like FEV1, required level of clinical care, and need for supplemental oxygen. Prospective studies should also evaluate the role of patient-specific factors like current or prior tobacco use and prior radiation on the development of irAEs. Finally prospective studies should aim to incorporate exploratory analyses of peripheral blood, bronchoscopy specimens and tumor samples to investigate potential predictive biomarkers that may indicate an increased risk for the development of pneumonitis. Several prospectitve studies evaluating alternative treatments for steroid-refractory irAEs have recently opened (NCT04438382; NCT04375228; NCT04552704). We eagerly await the results.

Infliximab is a commonly used immune modulator in patients with primary steroid-refracory ICI-induced irAEs, including ICI-induced pneumonitis. These retrospective studies, the largest published to date, demonstrated a high mortality rate (due either to progressive pneumonitis or 
infectious complications from infliximab) in patients with primary steroid-refractory ICI-induced pneumonitis who received infliximab. While patients with primary steroidrefractory ICI-induced pneumonitis have more severe disease than patients with recurrent ICI-induced pneumonitis, these studies raise serious questions about the efficacy of infliximab in treating steroid-refractory, ICIinduced pneumonitis. Of the 17 patients with primary steroid-refractory ICI-induced pneumonitis who received infliximab with high-dose steroids and/or another immunomodulatory agent (Balaji et $a l=0 / 5$; Beattie et $a l=3 / 12$ ), only $3(18 \%)$ showed durable clinical responses and all had died. This low treatment response rate with infliximab in primary steroid-refractory ICI-induced pneumonitis demonstrates the need for academically driven, prospective research on treatment algorithms for irAEs. Given that Balaji et al demonstrated that patients who received IVIG monotherapy had improved oxygen requirements, a reduced level of care, and reduced ICIinduced pneumonitis-related fatalies, IVIG should be considered as a reasonable alternative to infliximab until prospective irAE data are generated.

Twitter Margaret Gatti-Mays @DrGattiMays and James L Gulley @gulleyj1

Acknowledgements We would like to thank Bonnie Casey for her editing assistance.

Contributors Both authors composed, revised and edited this manuscript.

Funding The authors have not declared a specific grant for this research from any funding agency in the public, commercial or not-for-profit sectors.

Competing interests None declared.

Patient consent for publication Not required.

Provenance and peer review Commissioned; externally peer reviewed.
Open access This is an open access article distributed in accordance with the Creative Commons Attribution 4.0 Unported (CC BY 4.0) license, which permits others to copy, redistribute, remix, transform and build upon this work for any purpose, provided the original work is properly cited, a link to the licence is given, and indication of whether changes were made. See https://creativecommons.org/ licenses/by/4.0/.

\section{ORCID iDs}

Margaret Gatti-Mays http://orcid.org/0000-0001-8914-2897

James L Gulley http://orcid.org/0000-0002-6569-2912

\section{REFERENCES}

1 Puzanov I, Diab A, Abdallah K, et al. Managing toxicities associated with immune checkpoint inhibitors: consensus recommendations from the Society for immunotherapy of cancer (SITC) toxicity management Working group. J Immunother Cancer 2017;5:95

2 Nishino M, Giobbie-Hurder A, Hatabu H, et al. Incidence of programmed cell death 1 Inhibitor-Related pneumonitis in patients with advanced cancer: a systematic review and meta-analysis. JAMA Oncol 2016;2:1607-16.

3 Michot JM, Bigenwald C, Champiat S, et al. Immune-related adverse events with immune checkpoint blockade: a comprehensive review. Eur J Cancer 2016;54:139-48.

4 Wang DY, Salem J-E, Cohen JV, et al. Fatal toxic effects associated with immune checkpoint inhibitors: a systematic review and metaanalysis. JAMA Oncol 2018;4:1721-8.

5 Martins F, Sofiya L, Sykiotis GP, et al. Adverse effects of immunecheckpoint inhibitors: epidemiology, management and surveillance. Nat Rev Clin Oncol 2019;16:563-80.

6 Thompson JA, Schneider BJ, Brahmer J, et al. NCCN guidelines insights: management of immunotherapy-related toxicities, version 1.2020. J Natl Compr Canc Netw 2020;18:230-41.

7 Haanen JBAG, Carbonnel F, Robert C, et al. Management of toxicities from immunotherapy: ESMO clinical practice guidelines for diagnosis, treatment and follow-up. Ann Oncol 2017;28:iv119-42.

8 Postow MA, Hellmann MD. Adverse events associated with immune checkpoint blockade. N Engl J Med 2018;378:158-68.

9 Balaji A, Hsu M, Lin CT, et al. Steroid-refractory PD-(L)1 pneumonitis: incidence, clinical features, treatment, and outcomes. $J$ Immunother Cancer 2021;9:e001731.

10 Beattie J, Rizvi H, Fuentes P, et al. Success and failure of additional immune modulators in steroid-refractory/resistant pneumonitis related to immune checkpoint blockade. J Immunother Cancer 2021;9:e001884. 